TRANSACTIONS OF THE

AMERICAN MATHEMATICAL SOCIETY

Volume 170, August 1972

\title{
EXTREME LIMITS OF COMPACTA VALUED FUNCTIONS $\left({ }^{1}\right)$
}

BY

\author{
T. F. BRIDGLAND, JR.*
}

\begin{abstract}
Let $X$ denote a topological space and $\Omega(X)$ the space of all nonvoid closed subsets of $X$. Recent developments in analysis, especially in control theory, have rested upon the properties of the space $\Omega(X)$ where $X$ is assumed to be metric but not necessarily compact and with $\Omega(X)$ topologized by the Hausdorff metric. For a continuation of these developments, it is essential that definitions of extreme limits of sequences in $\Omega(X)$ be formulated in such a way that the induced limit is topologized by the Hausdorff metric. It is the purpose of this paper to present the formulation of such a definition and to examine some of the ramifications thereof. In particular, we give several theorems which embody "estimates of Fatou" for integrals of set valued functions.
\end{abstract}

0 . Let $X$ denote a topological space; the space of all nonvoid closed subsets of $X$ may be denoted by $2^{x}[1, \mathrm{p} .160]$ and the space of all nonvoid, compact subsets of $X$ by $\Omega(X)$. Definitions (contained in Definition 5 below) of extreme limits for sequences in $2^{x}$ have been given, and the ir consequences examined by Kuratowski ([1], [2]). Berge [3, p. $118 \mathrm{ff}$.] has extended these definitions and some of the consequences to filtered families of elements of $2^{x}$. A notion of limit of a sequence (or of a filtered family) is induced naturally by these definitions of extreme limits. As Kuratowski [2, p. 49] points out, if $X$ is compact metric then this induced limit in $2^{x}$ is topologized by the Hausdorff metric on $2^{x}$; on the other hand, Berge [3, p. 124] has pointed out that, in general, the induced limit in $2^{x}$ cannot be topologized. In particular, it is known [1, p. 342] that the induced limit in $2^{x}$ is not topologized by the Hausdorff metric when $X$ is metric but not compact.

Recent developments in analysis, especially in control theory (for example [4], [5], [6]), have rested upon the properties of the space $\Omega(X)$ where $X$ is assumed to be metric but not necessarily compact and with $\Omega(X)$ topologized by the Hausdorff metric. For a continuation of these developments it is essential that definitions of extreme limits of sequences (resp. filtered families) in $\Omega(X)$ be formulated in such a way that the induced limit is topologized by the Hausdorff metric. It is the purpose of this paper to present the formulation of such a

Received by the editors December 19, 1969.

AMS 1970 subject classifications. Primary 54C60, 28-00, 28A25; Secondary 49 E10.

Key words and phrases. Set valued functions, trajectory integral, control theory, multivalued functions, compacta valued functions, generalized differential equations.

(1) This research was sponsored by the National Science Foundation under grant GP-11543.

* This paper is being published posthumously. Professor Bridgland died on July 17, 1970 . 
definition and to examine some of the ramifications thereof. Expecially, noteworthy among these are Theorems 11 and 13 which embody "estimates of Fatou" for the trajectory integral [6] similar in nature to those obtained in the pioneer work of Aumann [7].

1. In the sequel, $X$ will denote, minimally, a metric space with metric denoted by $\rho$. The distance between a point $x \in X$ and a nonvoid subset, $A$, of $X$ will be denoted by $\gamma(x, A)$ :

$$
\gamma(x, A)=\inf \{\rho(x, a) \mid a \in A\} .
$$

The Hausdorff metric, $\omega$, on $\Omega(X)$ is defined by

$$
\omega(A, B)=\min \{\bar{\omega}(A, B), \bar{\omega}(B, A)\}
$$

where

$$
\bar{\omega}(A, B)=\sup \{\gamma(a, B) \mid a \in A\} .
$$

Let $S$ be a nonvoid subset of $R$, the set of real numbers( ${ }^{2}$ ), and let $A: S \rightarrow$ $\Omega(X)$. Let $t_{0} \in S^{\prime} \subset R^{*}$ where $R^{*}$ is the extended real number system and $S^{\prime}$ is the derived set of $S$; thus if $S=\Re$, the set of natural numbers, then $S^{\prime}=\{\infty\}$. Let $\varnothing$ denote the void set.

Definition 1. The upper cluster set, $\mathcal{U}\left(t_{0}, A\right)$, of $A$ at $t_{0}$ is defined by

$$
\mathcal{U}\left(t_{0}, A\right)=\left\{L \in \Omega(X) \mid \lim _{t \rightarrow t_{0}} \bar{\omega}(A(t), L)=0\right\} \text {; }
$$

the lower cluster set, $\mathcal{L}\left(t_{0}, A\right)$, of $A$ at $t_{0}$ is defined by

$$
\mathcal{L}\left(t_{0}, A\right)=\left\{L \in \Omega(X) \mid \lim _{t_{\rightarrow} t_{0}} \bar{\omega}(L, A(t))=0\right\} .
$$

Definition 2. The upper limit of $A$ at $t_{0}$ is defined by

$$
\begin{array}{ll}
\varlimsup_{\lim _{\rightarrow \rightarrow t_{0}} A(t)=\bigcap\left\{L \mid L \in \mathcal{U}\left(t_{0}, A\right)\right\},} \mathcal{U}\left(t_{0}, A\right) \neq \varnothing ; \\
\varlimsup_{t \rightarrow t_{0}} A(t)=X, & \mathcal{U}\left(t_{0}, A\right)=\varnothing .
\end{array}
$$

The lower limit of $A$ at $t_{0}$ is defined by

$$
\varliminf_{t \rightarrow t_{0}} A(t)=\operatorname{cl}\left(\bigcup\left\{L \mid L \in \mathscr{L}\left(t_{0}, A\right)\right\}\right) .
$$

Here cl $B$ denotes the closure of $B$.

As an immediate consequence of Definition 1 we obtain

Lemma 1. There exists $K \in \Omega(X)$ such that $\lim _{t \rightarrow t_{0}} \omega(A(t), K)=0$ if and only if $\mathcal{U}\left(t_{0}, A\right) \cap \mathfrak{L}\left(t_{0}, A\right) \neq \emptyset$; in this event $\mathcal{U}\left(t_{0}, A\right) \cap \mathfrak{L}\left(t_{0}, A\right)=\{K\}$.

Our next few results develop the properties of the extreme limits defined in Definition 2.

(2) Although with one exception (Theorem 15) we restrict our attention to functions with domains in $R$, our results generalize easily when $R$ is replaced by any Hausdorff space; the generalization to filtered families of sets is also easy. 
Lemma 2. $\underline{\lim }_{t \rightarrow t_{0}} A(t) \subset \varlimsup_{\lim _{t \rightarrow t_{0}}} A(t)$.

Proof. The assertion is trivial if either $\underline{\lim }_{t \rightarrow t_{0}} A(t)=\emptyset$ or $\overline{\lim }_{t \rightarrow t_{0}} A(t)=X$. Suppose both conditions fail; then $\mathscr{L}\left(t_{0}, A\right) \neq \varnothing \neq \mathcal{U}^{t}\left(t_{0}, A\right)$. If it can be shown that $K \subset L$ for all $(K, L) \in \mathfrak{L}\left(t_{0}, A\right) \times \mathcal{U}\left(t_{0}, A\right)$ then the remainder of the proof follows easily from Definition 2. We prove this assertion only for the case $t_{0} \neq \pm \infty$; the proof for the excluded cases is an easy modification of the one we shall give. Given $\epsilon>0$, it follows from Definition 1 that there exists $\delta=\delta\left(\epsilon, t_{0}\right)>0$ such that if $t \in S$ and $0<\left|t-t_{0}\right|<\delta$ then $\bar{\omega}(K, A(t))<\epsilon / 2$ and $\bar{\omega}(A(t), L)<\epsilon / 2$. By virtue of the "relaxed" triangle law [4, Lemma 1.1$]$ which is easily shown to hold in the present context, we have for $t \in S$ and $0<\left|t-t_{0}\right|<\delta$,

$$
\bar{\omega}(K, L) \leq \bar{\omega}(K, A(t))+\bar{\omega}(A(t), L)<\epsilon / 2+\epsilon / 2=\epsilon .
$$

Since $\epsilon$ was arbitrary there follows $\bar{\omega}(K, L)=0$.

Lemma 3. Let $\mathcal{L} \subset \Omega(X)$ be nonvoid and let $\left\{q_{n}\right\} \subset X$ satisfy $\lim _{n \rightarrow \infty} \gamma\left(q_{n^{\prime}} L\right)$ $=0$ for each $L \in \mathscr{L}$; then $\left\{q_{n}\right\}$ bas a limit point in $\bigcap\{L \mid L \in \mathscr{Q}\}$.

Proof. Let $L \in \mathscr{\&}$ be arbitrary but fixed; let $l_{n} \in L$ be a point nearest $q_{n}$. Since $L$ is compact, $\left\{l_{n}\right\}$ has a limit point $l \in L$ and it is easy to see that a subsequence, $\left\{q_{n_{k}}\right\}$, of $\left\{q_{n}\right\}$ converges to $l$. If $\mathscr{L}=\{L\}$ we are through; otherwise, if $M$ is any other element of $\mathcal{L}$, let $m_{k} \in M$ be a point nearest $q_{n_{k}}$. Then $\left\{m_{k}\right\}$ has a limit point $m \in M$ and clearly $m=l$.

Corollary 1. $\overline{\lim }_{t \rightarrow t_{0}} A(t) \neq \varnothing$.

Proof. The assertion is obvious if $\mathcal{U}\left(t_{0}, A\right)=\emptyset$; hence let $L \in \mathcal{U}\left(t_{0}, A\right)$ and let $\left\{t_{n}\right\} \subset S$ and $\left\{q_{n}\right\}$ satisfy $t_{n} \neq t_{0}, t_{n} \rightarrow t_{0}$ and $q_{n} \in A\left(t_{n}\right)$. Since $\gamma\left(q_{n^{\prime}} L\right) \leq$ $\bar{\omega}\left(A\left(t_{n}\right), L\right)$ there follows $\lim _{n \rightarrow \infty} \gamma\left(q_{n}, L\right)=0$; by Lemma $3,\left\{q_{n}\right\}$ has a limit point in $\varlimsup_{t \rightarrow t_{0}} A(t)$.

In the light of Corollary 1 and Lemma 2 we may formulate the following

Definition 3. If $\underline{\lim }_{t \rightarrow t_{0}} A(t)=\varlimsup_{\lim _{t \rightarrow t_{0}}} A(t)$ we shall say that $A$ bas a limit $L$ at $t_{0}$ and define $L$ to be the common value of the se extreme limits; we write briefly $\lim _{t \rightarrow t_{0}} A(t)=L$. When $\underline{\lim }_{t \rightarrow t_{0}} A(t)$ is noncompact (which can happen only when $X \notin \Omega(X))$ we may indicate this by writing $\lim _{t \rightarrow t_{0}} A(t)=X$. This convention is justified by observing that in this case we already have $\overline{\lim }_{t \rightarrow t_{0}} A(t)$ $=X$ by virtue of Lemma 2 and Definition 2 .

Theorem 1. Let $K \in \Omega(X)$. In order that $K \in \mathcal{U}\left(t_{0}, A\right)$ it is necessary and sufficient that $\varlimsup_{\lim _{t \rightarrow t_{0}}} A(t) \subset K ;$ moreover, in this event $\varlimsup_{\lim _{t \rightarrow t_{0}}} A(t) \in \Omega(X)$.

Proof. The final assertion of the theorem is a consequence of the following facts: by Definition 2, $\varlimsup_{t \rightarrow t_{0}} A \cdot(t)$ is closed and, by Corollary 1, nonvoid; by 
the first part of the theorem, it is a subset of a compact set. Turning our attention to the first part of the theorem we observe that the necessity of the condition is a trivial consequence of Definition 2. We shall prove the contrapositive of the sufficiency. Thus we suppose $K \notin U\left(t_{0}, A\right)$; we must show that $\varlimsup_{\lim _{t \rightarrow t_{0}}} A(t) \not \subset K$. If $\mathcal{U}\left(t_{0}, A\right)=\emptyset$ this is trivial; hence we suppose also that $\mathcal{U}\left(t_{0}, A\right) \neq \varnothing$. By our assumption on $K$ there exist $\epsilon>0$ and sequences $\left\{t_{n}\right\},\left\{q_{n}\right\}$ such that $t_{n} \neq t_{0}$, $t_{n} \rightarrow t_{0}, q_{n} \in A\left(t_{n}\right)$ and

$$
\gamma\left(q_{n}, K\right)=\bar{\omega}\left(A\left(t_{n}\right), K\right) \geq \epsilon .
$$

For any $L \in \mathcal{U}\left(t_{0}, A\right), \lim _{n \rightarrow \infty} \gamma\left(q_{n}, L\right) \leq \lim _{n \rightarrow \infty} \bar{\omega}\left(A\left(t_{n}\right), L\right)=0$; hence by Lemma 3, $\left\{q_{n}\right\}$ has a limit point $l \in \overline{\lim }_{t \rightarrow t_{0}} A(t)$. Consequently, if $\left\{q_{n_{k}}\right\}$ is a subsequence, converging to $l$, of $\left\{q_{n}\right\}$ then for sufficiently large values of $k$

$$
\gamma(l, K) \geq \gamma\left(q_{n_{k}}, K\right)-\rho\left(q_{n_{k}}, l\right)>\epsilon-\epsilon / 2=\epsilon / 2 .
$$

Theorem 2. Let $K \in \Omega(X)$. In order that $K \in \mathfrak{I}\left(t_{0}, A\right)$ it is necessary and sufficient that $K \subset \underline{\lim }_{t_{t \rightarrow t_{0}}} A(t)$.

Proof. The necessity of the condition is a trivial consequence of Definition 2. We shall prove the contrapositive of the sufficiency. Thus we suppose that $K \notin$ $\mathcal{L}\left(t_{0}, A\right)$; we must show that $K \not \subset \underline{\lim }_{t_{\rightarrow} t_{0}} A(t)$. If $\mathcal{L}\left(t_{0}, A\right)=\emptyset$ this is trivial;

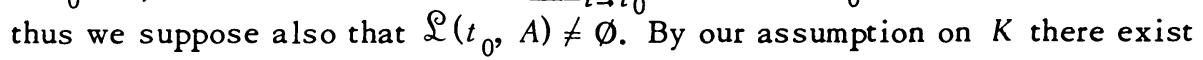
$\epsilon>0$ and sequences $\left\{t_{n}\right\},\left\{k_{n}\right\}$ such that $t_{n} \neq t_{0}, t_{n} \rightarrow t_{0}, k_{n} \in K$ and $\gamma\left(k_{n}, A\left(t_{n}\right)\right)>\epsilon$. Since $K \in \Omega(X)$ we may assume without loss of generality that $\left\{k_{n}\right\}$ converges to a point $k \in K$. Then

$$
\gamma\left(k_{n}, A\left(t_{n}\right)\right) \leq \rho\left(k_{n}, k\right)+\gamma\left(k, A\left(t_{n}\right)\right)
$$

so that $\lim _{n \rightarrow \infty} \gamma\left(k, A\left(t_{n}\right)\right) \geq \epsilon$. Now for each $L \in \mathscr{P}\left(t_{0}, A\right)$ there exists $\delta=$ $\delta\left(\epsilon, t_{0}, L\right)>0$ such that $\bar{\omega}(L, A(t))<\epsilon / 4$ for all $t$ satisfying $0<\left|t-t_{0}\right|<\delta$; in particular, for $n$ sufficiently large, $\bar{\omega}\left(L, A\left(t_{n}\right)\right)<\epsilon / 2$. Hence for $n$ sufficiently large

$$
\gamma(k, L) \geq \gamma\left(k, A\left(t_{n}\right)\right)-\bar{\omega}\left(L, A\left(t_{n}\right)\right)>\epsilon-\epsilon / 2=\epsilon / 2 .
$$

Suppose $\xi \in \bigcup\left\{L \mid L \in \mathscr{L}\left(t_{0}, A\right)\right\}$; then there exists $L_{\xi} \in \mathfrak{I}\left(t_{0}\right.$, A) such that $\xi \in L_{\xi}$ and then

$$
\rho(k, \xi) \geq \gamma\left(k, L_{\xi}\right)>\epsilon / 2 .
$$

If $\zeta \in \underline{\lim }_{t \rightarrow t_{0}} A(t)$ then there exist $\left\{\xi_{n}\right\} \subset \bigcup\left\{L \mid L \in \mathscr{L}\left(t_{0}, A\right)\right\}$ and $\left\{L_{n}\right\} \subset$ $\mathfrak{L}\left(t_{0}, A\right)$ such that $\xi_{n} \rightarrow \zeta$ and $\xi_{n} \in L_{n}$; hence for sufficiently large values of $n$ we find

$$
\rho(k, \zeta) \geq \rho\left(k, \xi_{n}\right)-\rho\left(\zeta, \xi_{n}\right) \geq \gamma\left(k, L_{n}\right)-\rho\left(\zeta, \xi_{n}\right)>\epsilon / 2-\epsilon / 4=\epsilon / 4 .
$$


Thus $\gamma\left(k, \underline{\lim }_{t \rightarrow t_{0}} A(t)\right) \geq \epsilon / 4$ and the proof is complete.

Our next result shows that the limit concept defined in Definition 3 is correct in the sense that it is topologized by the Hausdorff metric on $\Omega(X)$. It is an immediate consequence of Lemma 1 together with Theorems 1 and 2 .

Theorem 3. Let $K \in \Omega(X)$; then $\lim _{t \rightarrow t_{0}} \omega(A(t), K)=0$ if and only if $\lim _{t \rightarrow t_{0}} A(t)=K$.

A classical theorem of real analysis asserts $[8,7.6 .2]$ that a monotone realvalued function defined on a closed interval $S \subset R$ is regulated on $S$. As an application of the foregoing results we shall prove the analog of this theorem for functions with range in $\Omega(X)$. First we must make precise the notions of monotonicity and regulatedness for such functions. If we define

$$
\mathcal{U}^{+}\left(t_{0}, A\right)=\left\{L \in \Omega(X) \mid \lim _{t \rightarrow t_{0}+0} \bar{\omega}(A(t), L)=0\right\}
$$

with corresponding definitions of $\mathcal{U}^{-}\left(t_{0}, A\right), \mathfrak{L}^{+}\left(t_{0}, A\right)$ and $\mathfrak{L}^{-}\left(t_{0}, A\right)$ then the concept of one-sided limits corresponding to Definition 3 has an obvious formulation.

Definition 4. If $F: S \rightarrow \Omega(X)$ satisfies $\bar{\omega}\left(F\left(t_{1}\right), F\left(t_{2}\right)\right)=0$ for all $t_{1}, t_{2} \in S$ such that $t_{1}<t_{2}$ then $F$ is (monotone) nondecreasing on $S$; if $\bar{\omega}\left(F\left(t_{2}\right), F\left(t_{1}\right)\right)=0$ for all such $t_{1}, t_{2} \in S$ then $F$ is (monotone) nonincreasing on $S$. If $S$ is a closed interval and $F$ has one-sided limits at each point of $S$ then $F$ is regulated on $S$.

Theorem 4. If $S \subset R$ is a closed interval and $F: S \rightarrow \Omega(X)$ is monotone on $S$ then $F$ is regulated on $S$.

Proof. We give the proof for $F$ nondecreasing on $S$; the proof for $F$ nonincreasing is similar. Let $t_{0} \in S$ be fixed; it is easy to see that $F\left(t_{0}\right) \in \mathscr{L}^{+}\left(t_{0}, F\right)$ and that $F(t) \in \mathcal{U}^{+}\left(t_{0}, F\right)$ for all $t \in S$ such that $t>t_{0}$. Hence by Theorem 1 , $\overline{\lim }_{t \rightarrow t_{0}+0} F(t) \in \Omega(X)$ and

$$
\varlimsup_{t \rightarrow t_{0}+0} F(t) \equiv \bigcap\left\{L \mid L \in \mathcal{U}^{+}\left(t_{0}, F\right)\right\} \subset \bigcap\left\{F(t) \mid t \in S \cap\left(t_{0}, \infty\right)\right\} .
$$

Now if $x \in \bigcap \cdot\left\{F(t) \mid t \in S \cap\left(t_{0}, \infty\right)\right\}$ then $\gamma(x, F(t))=0$ for $t \in S \cap\left(t_{0}, \infty\right)$; hence $\{x\} \in \mathscr{L}^{+}\left(t_{0}, F\right)$ so that, by Theorem $2, x \in \underline{\lim }_{t \rightarrow t_{0}+0} F(t)$. But then

and we conclude

$$
\bigcap\left\{F(t) \mid t \in S \cap\left(t_{0}, \infty\right)\right\} \subset \underset{t \rightarrow t_{0}+0}{\lim _{1}} F(t)
$$

$$
F\left(t_{0}+0\right) \equiv \lim _{t \rightarrow t_{0}+0} F(t)=\bigcap\left\{F(t) \mid t \in S \cap\left(t_{0}, \infty\right)\right\} \supset F\left(t_{0}\right)
$$

Similarly, it is easy to see that $F\left(t_{0}\right) \in \mathcal{U}^{-}\left(t_{0}, F\right)$ and that $F(t) \in \mathscr{L}^{-}\left(t_{0}, F\right)$ for all $t \in S \cap\left(-\infty, t_{0}\right)$. Therefore 


$$
\begin{array}{r}
\operatorname{cl}\left(\bigcup\left\{F(t) \mid t \in S \cap\left(-\infty, t_{0}\right)\right\}\right) \subset \operatorname{cl}\left(\bigcup\left\{L \mid L \in \mathcal{L}^{-}\left(t_{0}, F\right)\right\}\right) \\
\subset \underset{t \rightarrow t_{0}-0}{\frac{\lim }{t_{0}}} F(t) \subset \varlimsup_{t \rightarrow t_{0}-0} F(t) \in \Omega(X) .
\end{array}
$$

But $\bar{\omega}\left(F(t), \operatorname{cl}\left(\bigcup\left\{F(\tau) \mid \tau \in S \cap\left(-\infty, t_{0}\right)\right\}\right)=0\right.$ for $t \in S \cap\left(-\infty, t_{0}\right)$ so that $\operatorname{cl}\left(\bigcup\left\{F(t) \mid t \in S \cap\left(-\infty, t_{0}\right)\right\}\right) \in \mathcal{U}^{-}\left(t_{0}, F\right)$; hence, by Theorem 1 ,

$$
\varlimsup_{t \rightarrow t_{0}-0} F(t) \subset \operatorname{cl}\left(\bigcup\left\{F(t) \mid t \in S \cap\left(-\infty, t_{0}\right)\right\}\right) \text {. }
$$

We conclude that

$$
F\left(t_{0}-0\right) \equiv \lim _{t \rightarrow t_{0}-0} F(t)=\mathrm{cl}\left(\bigcup\left\{F(t) \mid t \in S \cap\left(-\infty, t_{0}\right)\right\}\right) \subset F\left(t_{0}\right) .
$$

Next we obtain a limited characterization of $\varlimsup_{t \rightarrow t_{0}} A(t)$ which will assume its primary importance when $X$ is compact. In that case the availability of this characterization will facilitate a comparison of the present concept of extreme limits with that used by Kuratowski and Berge. In the remainder of this section we shall restrict our attention to the case $t_{0} \neq \pm \infty$. As with the proof of Lemma 2 the excluded cases may be treated by means of an obvious modification of the treatment we shall give.

For $\delta>0$ let $Z(\delta)$ be defined by

$$
Z(\delta)=\operatorname{cl}\left(\bigcup\left\{A(t)|t \in S ; 0<| t-t_{0} \mid<\delta\right\}\right)
$$

and let $V\left(t_{0}, A\right)$ be defined by

$$
V\left(t_{0}, A\right)=\bigcap\{Z(\delta) \mid \delta>0\} .
$$

Now for $0<\delta_{1}<\delta_{2}, Z\left(\delta_{1}\right) \subset Z\left(\delta_{2}\right)$ and from this it follows that if $\bar{\delta}>0$ is fixed and $\left\{\delta_{n}\right\}$ is a positive monotone null sequence satisfying $\delta_{1} \leq \bar{\delta}$ then

$$
V\left(t_{0}, A\right)=\bigcap\{Z(\delta) \mid 0<\delta \leq \bar{\delta}\}=\bigcap\left\{Z\left(\delta_{n}\right) \mid n \in \Re\right\} .
$$

Hence, by [4, Theorem 1.6], if $Z(\bar{\delta}) \in \Omega(X)$ then $V\left(t_{0}, A\right) \in \Omega(X)$ and

$$
\lim _{n \rightarrow \infty} \bar{\omega}\left(Z\left(\delta_{n}\right), V\left(t_{0}, A\right)\right)=0
$$

The desired characterization of $\overline{\lim }_{t \rightarrow t_{0}} A(t)$ is contained in

Theorem 5. If $Z(\bar{\delta}) \in \Omega(X)$ for some $\bar{\delta}>0$ then

$$
\varlimsup_{t \rightarrow t_{0}} A(t)=V\left(t_{0}, A\right) \in \Omega(X) .
$$

In order to prove Theorem 5 we shall need the following result.

Lemma 4. Let $Z(\bar{\delta}) \in \Omega(X)$ for some $\bar{\delta}>0$ and let $L \in \Omega(X)$. In order that $L \in \mathcal{U}\left(t_{0}, A\right)$ it is necessary and sufficient that $V\left(t_{0}, A\right) \subset L$; in this event $V\left(t_{0}, A\right) \in \mathcal{U}\left(t_{0}, A\right)$.

Proof (Necessity). Given $\epsilon>0$ there exists $\delta=\delta\left(\epsilon, t_{0}, L\right)>0$ such that 
$t \in S$ and $0<\left|t-t_{0}\right|<\delta$ imply that $\bar{\omega}(A(t), L)<\epsilon$. Let $\zeta \in Z\left(\delta^{\prime}\right)$ where $\delta^{\prime}=$ $\min \{\delta, \bar{\delta}\}$; then there exists $\left\{\xi_{n}\right\}$ satisfying $\lim _{n \rightarrow \infty} \xi_{n}=\zeta$ and $\xi_{n} \epsilon$ $\bigcup\left\{A(t)|t \in S ; 0<| t-t_{0} \mid<\delta^{\prime}\right\}$. Evidently $\gamma\left(\xi_{n}, L\right)<\epsilon$ for all $n \in \Re$ and then, since $\gamma(\cdot, L)$ is continuous, $\gamma(\zeta, L) \leq \epsilon$. Hence if $\zeta \in V\left(t_{0}, A\right)$ then $\gamma(\zeta, L) \leq \epsilon$ for all $\epsilon>0$ so that $\gamma(\zeta, L)=0$. Thus $V\left(t_{0}, A\right) \subset L$.

(Sufficiency). Let $\nu(\delta)$ be defined by

$$
\nu(\delta)=\bar{\omega}(Z(\delta), L), \quad 0<\delta \leq \bar{\delta}
$$

We observe that for $t \in S$ and $\left|t-t_{0}\right|<\delta$,

$$
\bar{\omega}(A(t), L) \leq \gamma(\delta) .
$$

$\nu$ is clearly nonnegative and nondecreasing; hence $\lim _{\delta \rightarrow 0} \nu(\delta)$ exists and is nonnegative. Let $\left\{\delta_{n}\right\}$ be a positive monotone null sequence with $\delta_{1}=\bar{\delta}$; then

$$
\nu\left(\delta_{n}\right) \leq \bar{\omega}\left(Z\left(\delta_{n}\right), V\left(t_{0}, A\right)\right)+\bar{\omega}\left(V\left(t_{0}, A\right), L\right) \equiv \bar{\omega}\left(Z\left(\delta_{n}\right), V\left(t_{0}, A\right)\right) .
$$

This estimate and $(*)$ imply that $\lim _{\delta \rightarrow 0} \nu(\delta)=0$ and then, by $(* *)$, $\lim _{t \rightarrow t_{0}} \bar{\omega}(A(t), L)=0$.

The proof of Theorem 5 is now easy. Under the hypothesis of that theorem and by virtue of Lemma $4, V\left(t_{0}, A\right) \in \mathcal{U}\left(t_{0}, A\right)$. Hence by Theorem $1 \overline{\lim }_{t_{\rightarrow} t_{0}} A(t)$ $\subset V\left(t_{0}, A\right)$. Again by Lemma $4, V\left(t_{0}, A\right) \subset L$ for all $L \in \mathcal{U}\left(t_{0}, A\right)$; hence $V\left(t_{0}, A\right) \subset \overline{\lim }_{t \rightarrow t_{0}} A(t)$.

2. In this section we examine the relationship between the present concept of extreme limits and that employed by Kuratowski and Berge. Definition 5 contains a formulation of the latter concept which is equivalent, in our context, to that given by Berge [3, p. 1191.

Definition 5. The lower limit, $\mathrm{Li}_{t \rightarrow t_{0}} A(t)$, of $A$ at $t_{0}$ is the set of all $x \in X$ for which $\lim _{n \rightarrow \infty} \gamma\left(x, A\left(t_{n}\right)\right)=0$ for every sequence $\left\{t_{n}\right\} \subset S-\left\{t_{0}\right\}$ satisfying $\lim _{n \rightarrow \infty} t_{n}=t_{0}$. The upper limit, Ls $t_{t \rightarrow t_{0}} A(t)$, of $A$ at $t_{0}$ is the set of all $x \in X$ for which $\lim _{n \rightarrow \infty} \gamma\left(x, A\left(t_{n}\right)\right)=0$ for at least one sequence $\left\{t_{n}\right\} \subset S-\left\{t_{0}\right\}$ satisfying $\lim _{n \rightarrow \infty} t_{n}=t_{0}$.

Our first result on the relationship between Definitions 2 and 5 is

Theorem 6. $\underline{\lim }_{t \rightarrow t_{0}} A(t)=\mathrm{Li}_{t \rightarrow t_{0}} A(t)$.

Proof. From Definitions 1 and 5 it follows that $x_{0} \in \mathrm{Li}_{t \rightarrow t_{0}} A(t)$ if and only if $\left\{x_{0}\right\} \in \mathscr{L}\left(t_{0}, A\right)$; hence, by Theorem $2, \mathrm{Li}_{t \rightarrow t_{0}} A(t) \subset \underline{\lim }_{t \rightarrow t_{0}} A(t)$. Similarly, since $L \in \mathscr{L}\left(t_{0}, A\right)$ and $x \in L$ imply $\{x\} \in \mathscr{L}\left(t_{0}, A\right)$, we find

$$
\bigcup\left\{L \mid L \in \mathscr{L}\left(t_{0}, A\right)\right\} \subset \underset{t \rightarrow t_{0}}{\operatorname{Li}_{i}} A(t)
$$

but, by [3, Corollary 1 , p. 121], $\mathrm{Li}_{t \rightarrow t_{0}} A(t)$ is closed so that Definition 2 yields $\lim _{t \rightarrow t_{0}} A(t) \subset \mathrm{Li}_{t \rightarrow t_{0}} A(t)$. 
Berge [3, p. 120] has given the following characterization of $\mathrm{Ls}_{t \rightarrow t_{0}} A(t)$.

Lemma 5. $\mathrm{Ls}_{t \rightarrow t_{0}} A(t)=V\left(t_{0}, A\right)$.

Since $Z(\delta) \in \Omega(X)$ for all $\delta>0$ when $X$ is compact, the next result is an immediate consequence of Theorem 5 and Lemma 5 .

Theorem 7. If $X$ is compact then $\varlimsup_{\lim _{t \rightarrow t_{0}}} A(t)=\mathrm{Ls}_{t \rightarrow t_{0}} A(t)$.

In the proof of the necessity of the condition of Lemma 4 we made no use of the condition " $Z(\delta) \in \Omega(X)$ ". Hence that proof, together with Lemma 5, shows that $\operatorname{Ls}_{t \rightarrow t_{0}} A(t) \subset \varlimsup_{\lim _{t \rightarrow t_{0}}} A(t)$ with $X$ just metric. If no further restriction is imposed on $X$ this inclusion may be proper, as exemplified when $X=R$ by the sequence $\left\{a_{n}\right\}$ defined by $a_{n}=\{0\}$ for $n$ even, $a_{n}=\{(n-1) / 2\}$ for $n$ odd. For we have $\operatorname{Ls}{ }_{n \rightarrow \infty} a_{n}=\{0\}$ whereas $\overline{\lim }_{n \rightarrow \infty} a_{n}=R$. If $X=E^{n}$, euclidean $n$-space, and $\overline{\lim }_{t \rightarrow t_{0}} A(t) \in \Omega(X)$, equality of the upper limits again ensues. Indeed, by Theorem $1, \varlimsup_{\lim _{t \rightarrow t_{0}}} A(t) \in \mathcal{U}\left(t_{0}, A\right)$ and then from Definition 1 there follows the fact that $Z(\delta)$ is bounded, hence compact, for $\delta$ sufficiently small; Theorem 5 thus applies. That $\overline{\lim }_{t \rightarrow t} A(t) \in \Omega(X)$ is unnecessary for equality is shown in $E^{1}$ by the example $A(t)=[-t, t]$; for in this case $\operatorname{Ls}_{t \rightarrow \infty} A(t)=\varlimsup_{t \rightarrow \infty} A(t)=E^{1}$.

The relationship between the two concepts of extreme limits under discussion here assumes its greatest importance when $X$ is compact, for in this case $\Omega(X) \equiv 2^{x}$. An illustration of this importance arises in connection $w$ ith the concept of semicontinuity of a function $A: S \rightarrow \Omega(X)$. Kuratowski has given one definition of semicontinuity for which, in the interest of conservation of (the author's) effort, we refer the reader to [2, p. 57]. The definitions of two other types of semicontinuity, one suggested by Berge [3, p. 120], are contained in the following definition.

Definition 6. $A$ is upper semicontinuous with respect to inclusion (usci) at $t_{0}$ if and only if $\lim _{t \rightarrow t_{0}} \bar{\omega}\left(A(t), A\left(t_{0}\right)\right)=0$. A is lsci at $t_{0}$ if and only if $\lim _{t \rightarrow t_{0}} \bar{\omega}\left(A\left(t_{0}\right), A(t)\right)=0$. A is upper semicontinuous in the sense of Berge $(u s c b)$ at $t_{0}$ if and only if Ls $\operatorname{tr}_{0} A(t) \subset A\left(t_{0}\right) . A$ is $l s c b$ at $t_{0}$ if and only if $A\left(t_{0}\right) \subset$ $\mathrm{Li}_{t \rightarrow t_{0}} A(t)$.

For semicontinuity in the sense of Kuratowski, cited above, we shall use the notation usck, lsck.

Before comparing these three notions of semicontinuity let us prove

Theorem 8. $A$ is usci at $t_{0}$ if and only if $\overline{\lim }_{t \rightarrow t_{0}} A(t) \subset A\left(t_{0}\right)$; it is lsci at $t_{0}$ if and only if $A\left(t_{0}\right) \subset \underline{\lim }_{t \rightarrow t_{0}} A(t)$.

Proof. If $\overline{\lim }_{t \rightarrow t_{0}} A(t) \subset A\left(t_{0}\right)$ then, by Theorem $1, A\left(t_{0}\right) \in \mathcal{U}\left(t_{0}, A\right)$ and this implies $A$ is usci at $t_{0}$. Conversely, $A$ being usci at $t_{0}$ implies $A\left(t_{0}\right) \in \mathcal{U}\left(t_{0}, A\right)$; again Theorem 1 permits the conclusion that $\overline{\lim }_{t \rightarrow t_{0}} A(t) \subset A\left(t_{0}\right)$. The proof for 
the second part of the theorem is analogous, with Theorem 2 being used in place of Theorem 1.

Our next result is an immediate consequence of Theorems 6,7 , and 8 .

Corollary 2. A is lsci at $t_{0}$ if and only if it is lscb at $t_{0}$. If $X$ is compact, $A$ is usci at $t_{0}$ if and only if it is uscb at $t_{0}$.

Kuratowski [2, pp. 61-62] has proved the following result.

Theorem 9. $A$ is lsck at $t_{0}$ if and only if it is lscb at $t_{0}$. If $X$ is compact, $A$ is usck at $t_{0}$ if and only if it is uscb at $t_{0}$.

Thus it is seen that, without restriction on the metric space $X$, the three types of lower semicontinuity are equivalent and that, if $X$ is compact, the three types of upper semicontinuity are equivalent.

3. Throughout this section we shall assume that $X$ is a Banach space with norm denoted by $\|\cdot\|$. We denote by $\Gamma(X)$ the space of nonvoid, compact convex subsets of $X$. Let $A: S \rightarrow \Gamma(X)$ and define

$$
\tilde{U}\left(t_{0}, A\right)=\mathcal{U}\left(t_{0}, A\right) \cap \Gamma(X) ; \quad \widetilde{\mathscr{L}}\left(t_{0}, A\right)=\mathscr{L}\left(t_{0}, A\right) \cap \Gamma(X) .
$$

Definition 7. The convex upper limit of $A$ at $t_{0}$ is defined by

$$
\begin{array}{ll}
\overline{\overline{\lim }} A(t)=\bigcap\left\{L \mid L \in \tilde{U}\left(t_{0}, A\right)\right\}, & \tilde{U}\left(t_{0}, A\right) \neq \varnothing ; \\
\overline{\overline{\lim _{t \rightarrow t_{0}}}} A(t)=X, & \tilde{U}\left(t_{0}, A\right) \neq \varnothing .
\end{array}
$$

The convex lower limit of $A$ at $t_{0}$ is defined by

$$
\underbrace{\lim _{t-t_{0}}}_{t \rightarrow t_{0}} A(t)=\operatorname{clco}\left(\bigcup\left\{L \mid L \in \widetilde{\mathscr{L}}\left(t_{0}, A\right)\right\}\right) \text {. }
$$

Here co $B$ and clco $B$ denote, respectively, the convex hull of $B$ and the closed convex hull of $B$; we adopt the view that $\varnothing$ is convex. Recall that, for $K \subset X$, clco $K=\operatorname{cl}(\operatorname{co} K)$.

We shall develop the properties of the extreme limits defined in Definition 7. Preliminarily we shall need Lemma 7 whose proof depends on the following paraphrase of a theorem of Mazur [9, V.2.6].

Lemma 6. If $K \in \Omega(X)$ then clco $K \in \Gamma(X)$.

Lemma 7. If $K \in \Omega(X)$ and $B \in \Gamma(X)$ then $\bar{\omega}(\operatorname{clco} K, B)=\bar{\omega}(K, B)$.

Proof. At the outset we have

$$
\bar{\omega}(K, B) \leq \bar{\omega}(K, \operatorname{clco} K)+\bar{\omega}(\operatorname{clco} K, B)=\bar{\omega}(\operatorname{clco} K, B) .
$$

For the reverse inequality, let $x \in$ co $K$; then by Carathéodory's theorem there 
exist $x_{i} \in K, i=1, \cdots, n+1$, and $\alpha_{i} \geq 0, i=1, \cdots, n+1$, such that $\sum_{i=1}^{n+1} \alpha_{i}=1$ and $x=\sum_{i=1}^{n+1} \alpha_{i} x_{i}$. Hence

$$
\gamma(x, B)=\gamma\left(\sum_{i=1}^{n+1} a_{i} x_{i}, B\right) \leq \sum_{i=1}^{n+1} a_{i} \gamma\left(x_{i}, B\right) \leq \bar{\omega}(K, B),
$$

the first inequality being a consequence of the convexity of the function $\gamma(\cdot, B)$ [10, Theorem 3.4, p. 35]. Thus if $\xi \epsilon \operatorname{clco} K=\operatorname{cl}(\operatorname{co} K)$ then by this last estimate and the continuity of $\gamma(\cdot, B), \gamma(\xi, B) \leq \bar{\omega}(K, B)$. Hence $\bar{\omega}(\operatorname{clco} K, B) \leq \bar{\omega}(K, B)$.

Although we shall not need it in the sequel, it is interesting to note the following generalization of [6, Lemma 1.3(ii)]: if $B, C \in \Omega(X)$ then $\bar{\omega}$ (clco $B$, clco $C$ ) $\leq \bar{\omega}(B, C)$.

Now we are in a position to examine the relationship between the extreme limits defined in Definitions 2 and 7.

Theorem 10. (i) clco $\left[\overline{\lim }_{t_{\rightarrow} t_{0}} A(t)\right]=\overline{\overline{\lim }}_{t_{\rightarrow} t_{0}} A(t)$;

(ii) $\underline{\lim }_{t \rightarrow t_{0}} A(t)=\operatorname{clco}\left[\underline{\lim }_{t_{\rightarrow} t_{0}} A(t)\right]$.

Proof. For the proof of (i) we observe that since $\tilde{U}\left(t_{0}, A\right) \subset \mathcal{U}\left(t_{0}, A\right)$, $\overline{\lim }_{t_{\rightarrow} t_{0}} A(t) \subset \overline{\overline{\lim }}_{t_{\rightarrow} t_{0}} A(t)$, equality holding if $\mathcal{U}\left(t_{0}, A\right)=\emptyset$. If $\mathcal{U}\left(t_{0}, A\right) \neq \varnothing$ then, by Theorem $1, \varlimsup_{\lim _{t \rightarrow t_{0}}} A(t) \in \mathcal{U}\left(t_{0}, A\right)$; hence, by Lemma 6 and Theorem 1 , $\operatorname{clco}\left[\overline{\lim }_{t_{\rightarrow} t_{0}} A(t)\right] \in \tilde{\mathcal{U}}\left(t_{0}, A\right)$. But then, by Definition $7, \overline{\overline{\lim }}_{t_{\rightarrow} t_{0}} A(t) \subset$ clco $\left[\overline{\lim }_{t_{\rightarrow} t_{0}} A(t)\right]$ and the proof of (i) is complete.

For the proof of (ii), since (a) $\mathfrak{L}\left(t_{0}, A\right) \subset \mathscr{L}\left(t_{0}, A\right)$ the validity of (ii) is trivial if $\mathscr{L}\left(t_{0}, A\right)=\emptyset$. Suppose $L \in \mathscr{L}\left(t_{0}, A\right)$ so that, by Lemma 7 , clco $L \epsilon$ $\mathcal{L}\left(t_{0}, A\right)$. This implies that

$$
\bigcup\left\{L \mid L \in \mathscr{L}\left(t_{0}, A\right)\right\}=\bigcup\left\{\text { clco } L \mid L \in \mathscr{L}\left(t_{0}, A\right)\right\}
$$

whereas inclusion (a) implies that

$$
\bigcup\left\{L \mid L \in \mathfrak{L}\left(t_{0}, A\right)\right\} \subset \bigcup\left\{L \mid L \in \mathfrak{L}\left(t_{0}, A\right)\right\} .
$$

Since clearly

$$
\bigcup\left\{\text { clco } L \mid L \in \mathscr{L}\left(t_{0}, A\right)\right\} \subset \bigcup\left\{L \mid L \in \mathfrak{L}\left(t_{0}, A\right)\right\},
$$

we obtain from the last three formula lines

$$
\bigcup\left\{\text { clco } L \mid L \in \mathfrak{L}\left(t_{0}, A\right)\right\}=\bigcup\left\{L \mid L \in \mathfrak{L}\left(t_{0}, A\right)\right\}=\bigcup\left\{L \mid L \in \mathfrak{L}\left(t_{0}, A\right)\right\} .
$$

From the second equality (ii) follows immediately.

Corollary 3. There exists $K \in \Gamma(X)$ such that $\lim _{t_{\rightarrow} t_{0}} \omega(A(t), K)=0$ if and only if $\widetilde{\mathbb{U}}\left(t_{0}, A\right) \cap \widetilde{\complement}\left(t_{0}, A\right) \neq \varnothing$. In this event, $\widetilde{U}\left(t_{0}, A\right) \cap t^{t_{0}} \widetilde{\mathscr{L}}\left(t_{0}, A\right)=\{K\}$ and

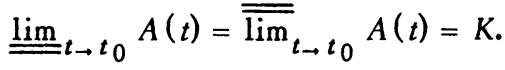


Corollary 4. $\overline{\overline{\lim }}_{t \rightarrow t_{0}} A(t) \neq \varnothing$.

Lemma 8. Let $K \in \Gamma(X)$. In order that $K \in \tilde{U}\left(t_{0}, A\right)$ it is necessary and sufficient that $\overline{\overline{\lim }}_{t_{\rightarrow} t_{0}} A(t) \subset K$; in this event ${\overline{\lim _{t_{\rightarrow} t_{0}}}} A(t) \in \Gamma(X)$.

Proof. The necessity of the condition is obvious. That the condition is sufficient follows from the first sentence of the proof of Theorem 10 together with Theorem 1. The final assertion follows from the first part of the theorem by virtue of Corollary 4.

4. In the interest of brevity and to conform to the notation of [6], in this section we set $\Omega\left(E^{n}\right)=\Omega^{n}, \Gamma\left(E^{n}\right)=\Gamma^{n}$ and $\Gamma\left(\mathcal{C}^{n}(I)\right)=\mathcal{K}^{n}(I)$ where $I$ is a compact interval in $R$ and $\mathcal{C}^{n}(I)$ is the Banach space of continuous functions on $I$ to $E^{n}$, normed by the supremum norm.

Lemma 9. $\Gamma^{n}$ is closed in $\Omega^{n}$.

Proof. Suppose, for $\left\{A_{m}\right\} \subset \Gamma^{n}$, that $\lim _{m \rightarrow \infty} A_{m}=A \in \Omega^{n}$; since $\bar{\omega}\left(A_{m},\{0\}\right)$ $\leq \bar{\omega}\left(A_{m}, A\right)+\bar{\omega}(A,\{0\})$ for all $m \in \mathcal{N},\left\{A_{m}\right\}$ is a bounded sequence. Thus by Blaschke's Auswahlsatz $\left\{A_{m}\right\}$ has a limit point in $\Gamma^{n}$; but since $\left\{A_{m}\right\}$ converges, this limit point is unique and coincides with $A$.

Definition 8. (i) A function $F: I \rightarrow \Omega^{n}$ is measurable if and only if the set $E(F, D)=\{t \in I \mid F(t) \cap D \neq \emptyset\}$ is Lebesgue measurable for each open set $D \subset E^{n}$.

(ii) Let $A$ be an index set and let $F_{\gamma}: I \rightarrow \Omega^{n}$ for each $\gamma \in A$; if there exists a Lebesgue summable function $b: I \rightarrow R$ such that $\bar{\omega}\left(F_{\gamma}(t),\{0\}\right) \leq b(t)$ for all $t \in I$ and all $\gamma \in A$ then $\left\{F_{\gamma} \mid \gamma \in A\right\}$ is uniformly integrably bounded. If $A=\{1\}$ the word "uniformly" is omitted.

(iii) Given $F: I \rightarrow \Omega^{n}$ let $\mathcal{F}_{I}(F)$ denote the set of all Lebesgue summable functions $u: I \rightarrow E^{n}$ having the property that $u(t) \in F(t)$ almost everywhere (a.e.) on $l$. Let $\mathcal{T}$ be defined by

$$
\left(\mathfrak{T}_{q}\right)(t)=\int_{0}^{t} q(\tau) d \tau, \quad t \in I
$$

the trajectory integral of $F, \mathcal{S}_{I}(F)$, is defined by

$$
\mathcal{S}_{I}(F)=\mathcal{J F}_{I}(F) \text {. }
$$

We shall establish counterparts (Theorems 11 and 13), for the trajectory integral, of Propositions 5.1 and 4.1, respectively, of [7]. These permit a proof of [6, Theorem 3.2] in the spirit of that given by Aumann for [7, Theorem 5].

Theorem 11. If $F_{k}: I \rightarrow \Gamma^{n}$ is measurable for eacb $k$ and $\left\{F_{k}\right\}$ is uniformly integrably bounded then $\mathcal{S}_{I}\left(\underline{\lim }_{k \rightarrow \infty} F_{k}\right) \subset \underline{\lim }_{k \rightarrow \infty} \mathcal{S}_{I}\left(F_{k}\right)$.

Proof. If $\mathcal{S}_{I}\left(\underline{\lim }_{k \rightarrow \infty} F_{k}\right)=\varnothing$ there is nothing to prove; hence, suppose $x \in \mathcal{S}_{I}\left(\underline{\lim }_{k \rightarrow \infty} F_{k}\right)$. Noting that $\mathcal{S}_{I}\left(F_{k}\right) \in K^{n}(I)[6$, Theorem 3.1$]$ for each $k$, 
we shall prove that $\{x\} \in \mathscr{L}\left(\infty,\left\{\mathcal{S}_{I}\left(F_{k}\right)\right\}\right)$. Now $\dot{x} \in \mathcal{F}_{I}\left(\underline{\lim }_{k \rightarrow \infty} F_{k}\right)$ so, since $F_{k}$ is measurable, by [6, Lemma 2.5] there exists $u_{k} \in \mathcal{F}_{I}\left(F_{k}\right)$ such that $u_{k}(t)$ is the point of $F_{k}(t)$ nearest $\dot{x}(t)$; evidently $\left\{u_{k}\right\}$ has the same uniform integrable bound as does $\left\{F_{k}\right\}$. We have

$$
\bar{\omega}\left(\{x\}, \mathcal{S}_{I}\left(F_{k}\right)\right) \equiv \gamma\left(x, \mathcal{S}_{I}\left(F_{k}\right)\right) \leq \int_{I}\left\|\dot{x}(\tau)-u_{k}(\tau)\right\| d \tau
$$

but $\left\|\dot{x}(t)-u_{k}(t)\right\|=\gamma\left(\dot{x}(t), F_{k}(t)\right) \equiv \bar{\omega}\left(\{\dot{x}(t)\}, F_{k}(t)\right)$ and, by Theorem 2 and the way $x$ was chosen, $\{\dot{x}(t)\} \in \mathscr{L}\left(\infty,\left\{F_{k}(t)\right\}\right)$ a.e. on $I$. If $b$ is the uniform integrable bound of $\left\{F_{k}\right\}$ then $\left\|\dot{x}(t)-u_{k}(t)\right\| \leq 2 \max \{\|\dot{x}(t)\|, b(t)\}$. Hence by the Lebesgue dominated convergence theorem, $\lim _{k \rightarrow \infty} \bar{\omega}\left(\{x\}, \mathcal{S}_{I}\left(F_{k}\right)\right)=0$.

Theorem 12. If $F_{k}: I \rightarrow \Omega^{n}$ is measurable for each $k$ and if $\overline{\lim }_{k \rightarrow \infty} F_{k}(t) \epsilon$ $\Omega^{n}$ on $I$ then the function $F: I \rightarrow \Omega^{n}$, defined by $F(t)=\varlimsup_{\lim _{k \rightarrow \infty}} F_{k}(t)$, is measurable on $I$.

Proof. In view of the discussion following Theorem 7, for all $t \in I, F(t)=$ $V\left(\infty,\left\{F_{k}(t)\right\}\right)$. Moreover, by virtue of the comments preceding Theorem 5 , we have $F(t)=\lim _{m_{\rightarrow} \infty} Z(m, t)$ where $Z(m, t)=\operatorname{cl}\left(\bigcup\left\{F_{k}(t) \mid k>m\right\}\right)$. It is easy to see that

$$
\{t \mid Z(m, t) \cap K \neq \varnothing\}=\bigcup\left\{\left\{t \mid F_{k}(t) \cap K \neq \varnothing\right\} \mid k>m\right\}
$$

hence, by hypothesis, $Z(m, \cdot)$ is measurable on $I$ for each $m$. It is then a consequence of $[6$, Theorem 2.1$]$ that $F$ is measurable on $I$.

Corollary 5. If $F_{k}: I \rightarrow \Gamma^{n}$ is measurable for each $k$ and $\left\{F_{k}\right\}$ is uniformly integrably bounded then $\mathcal{S}_{I}\left(\overline{\overline{\lim }}_{k \rightarrow \infty} F_{k}\right) \in \mathcal{K}^{n}(I)$.

Proof. Let $b$ be the integrable bound on $\left\{F_{k}\right\}$ and define $S_{b}(t)=\{x \mid\|x\| \leq$ $b(t)\}$; then, a.e. on $I, S_{b}(t) \in \tilde{U}\left(\infty,\left\{F_{k}(t)\right\}\right)$. Hence by Lemma $8, \overline{\lim }_{k_{\rightarrow \infty}} F_{k}(t) \subset$ $S_{b}(t) \in \Gamma^{n}$ a.e. on $I$. Denote by $E$ the set of points on which this last condition holds; then the Lebesgue measure of $I-E$ is zero. Now let us define functions $g: I \rightarrow E^{n}$ and $G_{k}: I \rightarrow \Gamma^{n}$ by

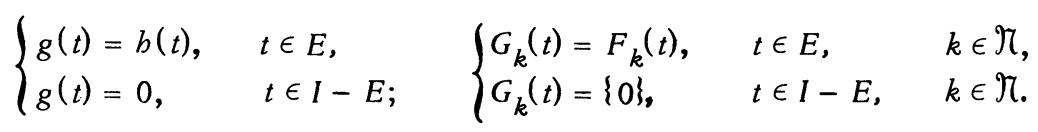

Then $\overline{\overline{\lim }}_{k \rightarrow \infty} G_{k}(t)=\overline{\overline{\lim }}_{k \rightarrow \infty} F_{k}(t)$ for $t \in E$ so that $\mathcal{S}_{I}\left(\overline{\overline{\lim }}_{k \rightarrow \infty} G_{k}\right)=$ $\mathcal{S}_{I}\left(\overline{\overline{\lim }}_{k \rightarrow \infty} F_{k}\right)$; moreover, $\overline{\overline{\lim }}_{k_{\rightarrow \infty}} G_{k}(t) \subset S_{g}(t) \in \Gamma^{n}$ for all $t \in I$. Consequently, if the function $t \rightarrow{\overline{\overline{\lim _{k \rightarrow \infty}}}}_{k_{k}} G_{k}(t)$ is measurable on $I$ then the conclusion of the the orem follows from [6, Theorem 3.1]. To establish this measurability, let $G$ be defined by $G(t)=\varlimsup_{\lim _{k \rightarrow \infty}} G_{k}(t)$; then, by Theorems 10 (i) and 12, $G$ is measurable on $I$. The requisite measurability now follows from the measurability of $G$ by virtue of Theorem $10(\mathrm{i})$ and [6, Corollary 2.1]. 
Theorem 13. If $F_{k}: I \rightarrow \Gamma^{n}$ is measurable for eacb $k$ and $\left\{F_{k}\right\}$ is uniformly integrably bounded, then

consequently $\overline{\overline{\lim }}_{k \rightarrow \infty} \mathcal{S}_{I}\left(F_{k}\right) \in \mathcal{K}^{n}(I)$.

$$
\overline{\overline{\lim }} \delta_{I}\left(F_{k}\right) \subset \mathcal{S}_{I}\left(\overline{\overline{\lim _{k \rightarrow \infty}}} F_{k}\right)
$$

Proof. By Corollary 5 and Lemma 8 the first assertion is equivalent to the assertion

$$
\varlimsup_{j \rightarrow \infty} \bar{\omega}\left(S_{I}\left(F_{j}\right), \mathcal{S}_{I}\left(\overline{\overline{\lim }} F_{k \rightarrow \infty}\right)\right)=0 .
$$

Now for each $j \in \Re$ there exists $\phi_{j} \in \mathcal{S}_{I}\left(F_{j}\right)$ such that

$$
\gamma\left(\phi_{j}, \mathcal{S}_{I}\left(\overline{\overline{\lim }} F_{k \rightarrow \infty}\right)\right)=\bar{\omega}\left(\mathcal{S}_{I}\left(F_{j}\right), \mathcal{S}_{I}\left(\overline{\overline{\lim }} F_{k}\right)\right)
$$

hence there exists a subsequence, which we denote by the same indices, of $\left\{\phi_{j}\right\}$ such that

$$
\lim _{j \rightarrow \infty} \gamma\left(\phi_{j}, \mathcal{S}_{I}\left(\overline{\overline{\lim }} F_{k \rightarrow \infty}\right)\right)=\varlimsup_{j \rightarrow \infty} \bar{\omega}\left(\mathcal{S}_{I}\left(F_{j}\right), \mathcal{S}_{I}\left(\overline{\overline{\lim }} F_{k}\right)\right) .
$$

Let $b$ be the integrable bound of $\left\{F_{k}\right\}$; since $\left\{\phi_{j}\right\}$ is bounded (by $\int_{I} b(\tau) d \tau$ ) and equicontinuous (as a consequence of $\left\|\phi_{j}\left(t_{2}\right)-\phi_{j}\left(t_{1}\right)\right\| \leq\left|\int_{t_{1}}^{t_{2}} b(\tau) d \tau\right|$ ) it has a limit point $\phi$. By virtue of the continuity of $\gamma\left(\cdot, \mathcal{S}_{I}\left(\overline{\lim }_{k \rightarrow \infty} F_{k}\right)\right)$ we may deduce that

$$
\lim _{j \rightarrow \infty} \gamma\left(\phi_{j}, \mathcal{S}_{I}\left(\overline{\overline{\lim }} F_{k \rightarrow \infty}\right)\right)=\gamma\left(\phi, \mathcal{S}_{I}\left(\overline{\overline{\lim }} F_{k \rightarrow \infty}\right)\right)
$$

There is no difficulty in establishing that $\left\|\phi\left(t_{2}\right)-\phi\left(t_{1}\right)\right\| \leq\left|\int_{t_{1}}^{t_{2}} b(\tau) d \tau\right|$ whence we conclude that $\phi$ is absolutely continuous. By virtue of [9, IV.8.9] we may assume without loss of generality that $\left\{\dot{\phi}_{j}\right\}$ converges weakly to $\dot{\phi}$. Aumann's argument [7, Proposition 4.1] is applicable, mutatis mutandis, yielding the conclusion that, a.e. on $I, \dot{\phi}(t)$ is a convex combination of the limit points of $\left\{\dot{\phi}_{j}(t)\right\}$. Now it is easy to deduce with the aid of Lemma 8 that $\lim _{j \rightarrow \infty} \gamma\left(\dot{\phi}_{j}(t), \overline{\overline{\lim }}_{k \rightarrow \infty} F_{k}(t)\right)=0$ a.e. on $I$. In view of the convexity of the function $\gamma\left(\cdot, \overline{\overline{\lim }}_{k \rightarrow \infty} F_{k}(t)\right)$ a.e. on $I$ (vide the proof of Lemma 7), we may assert that $\gamma\left(\dot{\phi}(t),{\overline{\overline{\lim _{k \rightarrow \infty}}}}_{k_{k}} F_{k}(t)\right)=0$ a.e. on $I$. Therefore, $\phi \in \mathcal{S}_{I}\left(\overline{\overline{\lim }}_{k \rightarrow \infty} F_{k}\right)$ and this fact, together with the last three formula lines, completes the proof of the assertion made at the outset. The second assertion of the theorem is a direct consequence of the first together with Corollaries 4 and 5.

Theorem 14 [6, Theorem 3.2]. If $F_{k}: I \rightarrow \Gamma^{n}$ is measurable for each $k$, if $\left\{F_{k}\right\}$ is uniformly integrably bounded and if $\lim _{k_{\rightarrow} \infty} F_{k}(t)=F(t) \in \Omega^{n}$ on $I$, then $\mathcal{S}_{I}(F)$ and $\mathcal{S}_{I}\left(F_{k}\right)$ are in $\mathcal{K}^{n}(I)$ for each $k$ and $\lim _{k \rightarrow \infty} \mathcal{S}_{I}\left(F_{k}\right)=\mathcal{S}_{I}(F)$. 
Proof. By Lemma 9, $F(t) \in \Gamma^{n}$ on $I$; by [6, Theorem 2.1], $F$ is measurable and it is easily seen to have the same integrable bound as $\left\{F_{k}\right\}$. Hence it follows from [6, Theorem 3.1] that $\mathcal{S}_{I}(F) \in \mathcal{K}^{n}(I)$ and $\mathcal{S}_{I}\left(F_{k}\right) \in \mathcal{K}^{n}(I)$ for each $k$. Thus by

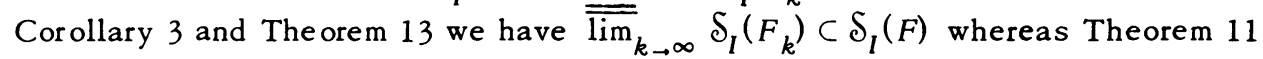
yields $\delta_{I}(F) \subset \underline{\lim }_{k \rightarrow \infty} \mathcal{S}_{I}\left(F_{k}\right)$. Together with Lemma 2 and Theorem 10(i), the last two formula lines yield the final assertion of the theorem.

Remark. Theorem 14 is actually a slight improvement on [6, The orem 3.2] due to the fact that in [6] the present Lemma 9 was overlooked.

As a final application of our methods we give a particularly transparent proof of a "measurable selection" theorem due to Castaing [11, Théorème 1].

Theorem 15. Let $D \subset E^{1} \times E^{n}$ be nonvoid and open and let $R: E^{1} \times E^{n} \rightarrow \Omega^{n}$ satisfy

(i) for each $t$ in the projection of $D$ on $E^{1}, R(t, \cdot)$ is usci on the set $D_{t}=$ $\left\{x \in E^{n} \mid(t, x) \in D\right\}$;

(ii) for each $x$ in the projection of $D$ on $E^{n}$ and each compact interval $I \subset E^{1}$ for which $I \times\{x\} \subset D, R(\cdot, x)$ is measurable on $I$.

If $I$ is a compact interval in $E^{1}$ and $S$ is a compact ball in $E^{n}$ satisfying $I \times S \subset D$ then for each continuous function $x: I \rightarrow S$ there exists a measurable function $\Lambda: I \rightarrow \Omega^{n}$ satisfying $\Lambda(t) \subset R(t, x(t))$ on $I$.

Proof. Let $s: I \rightarrow S$ be a step function; then, by (ii) and the argument for [6, Lemma 2.8], $R(\cdot, s(\cdot))$ is measurable on $I$. Now let $x: I \rightarrow S$ be continuous and let $\left\{s_{m}\right\}$ be a sequence of step functions converging uniformly on $I$ to $x$; without loss of generality we may suppose that $s_{m}: I \rightarrow S$. We shall show that a function $\Lambda$ possessing the requisite properties may be defined by

$$
\Lambda(t)=\varlimsup_{m \rightarrow \infty} R\left(t, s_{m}(t)\right)
$$

It is an easy consequence of Definition 1 that, for $t \in I, \mathcal{U}(x(t), R(t, \cdot)) \subset$ $\mathcal{U}\left(\infty,\left\{R\left(t, s_{m}(t)\right)\right\}\right)$; hence

$$
\Lambda(t) \subset \varlimsup_{x \rightarrow x(t)} R(t, x) \subset R(t, x(t)),
$$

the second inclusion being a consequence of (i) and Theorem 8 . The measurability of $\Lambda$ on $I$ is now immediate from Theorem 12.

Corollary 6. If in Theorem 15 the condition " $R: I \times E^{n} \rightarrow \Omega^{n}$ ", is replaced

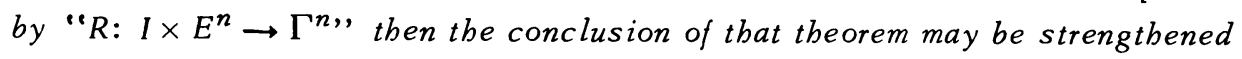
by replacing " $\Lambda: I \rightarrow \Omega^{n}$ " by " $\Lambda: I \rightarrow \Gamma^{n}$ ".

Proof. A function $\Lambda^{*}$ satisfying the restriction of this corollary is obtained by defining $\Lambda^{*}(t)=\operatorname{co}(\Lambda(t))$, where $\Lambda$ is the function whose existence is assured by Theorem 15 . That $\Lambda^{*}$ is measurable on $I$ then follows from that theorem together with [6, Corollary 2.1]. 


\section{REFERENCES}

1. K. Kuratowski, Topology. Vol. 1, Academic Press, New York; PWN, Warsaw, 1966. MR $36 \# 839$.

2. - Topology. Vol. 2, Academic Press, New York; PWN, Warsaw, 1968.

MR 41 \#467.

3. C. Berge, Topological spaces, Macmillan, New York, 1963.

4. T. F. Bridgland, Jr., Contributions to the theory of generalized differential equations. I, Math. Systems Theory 3 (1969), 17-50. MR 40 \#3018.

5. - Contributions to the theory of generalized differential equations. II, Math. Systems Theory 3 (1969), 156-165. MR 40 \#3018.

6. - Trajectory integrals of set valued functions, Pacific J. Math. 33 (1970), 43-68. MR 41 \#7061.

7. K. J. Aumann, Integrals of set-valued functions, j. Math. Anal. Appl. 12 (1965), 1-12. MR 32 \#2543.

8. J. Dieudonné, Foundations of modern analysis, Pure and Appl. Math., vol. 10, Academic Press, New York, 1960. MR 22 \#11074.

9. N. Dunford and J. T. Schwartz, Linear operators. I: General theory, Pure and Appl. Math., vol. 7, Interscience, New York, 1958. MR 22 \#8302.

10. F. A. Valentine, Convex sets, McGraw-Hill Series in Higher Math., McGraw-Hill, New York, 1964. MR 30 \#503.

11. C. Castaing, Sur les équations différentielles multivoques, C. R. Acad. Sci. Paris Sér. A-B 263 (1966), A63-A66. MR 34 \#397.

DEPARTMENT OF MATHEMATICS, DREXEL INSTITUTE OF TECHNOLOGY, PHILADELPHIA, PENNSYLVANIA 19104 